\title{
Geographical Distribution of Biomphalaria Snails in the State of Minas Gerais, Brazil
}

\author{
Cecília Pereira de Souza/ ${ }^{+}$, Roberta Lima Caldeira*, \\ Sandra Costa Drummond**, Alan Lane Melo***, Carlos Tito Guimarães, \\ Delza de Moura Soares, Omar dos Santos Carvalho*
}

\begin{abstract}
Laboratório de Malacologia *Laboratório de Helmintoses Intestinais, Centro de Pesquisas René RachouFiocruz, Av. Augusto de Lima 1715, 30190-002 Belo Horizonte, MG, Brasil **Fundação Nacional de Saúde, Belo Horizonte, MG, Brasil ***Departamento de Parasitologia, Universidade Federal de Minas Gerais, Belo Horizonte, MG, Brasil
\end{abstract}

Published and unpublished observations on geographical distribution of Biomphalaria snails in the State of Minas Gerais, Brazil, were compiled. This work is aimed at knowing the present occurrence of Biomphalaria species in this region, and at contributing to the elaboration of the planorbid chart of Minas Gerais. In malacological surveys, performed by several researchers, the presence of seven species of this genus was recorded. Those planorbids were found in 12 mesoregions, in $283(33.1 \%) \mathrm{mu-}$ nicipalities out of 853 with the following distribution: B. glabrata (185 municipalities), B. straminea (125), B. tenagophila (58), B. peregrina (57), B. schrammi (26), B. intermedia (20) and B. occidentalis (2). B. glabrata and B. tenagophila are found naturally infected by Schistosoma mansoni in Minas Gerais. In 24 municipalities the three snail hosts of S. mansoni in Brazil, B. glabrata, B. tenagophila and B. straminea, are present.

Key words: Biomphalaria - planorbids - geographic distribution - Minas Gerais - Brazil

Brazil is an endemic focus of schistosomiasis mansoni with over 6 million people infected (Rey 1991). The current national prevalence of schistosomiasis in Brazil indicates that it is still considered an important endemic parasitosis (Katz \& Peixoto 2000). The endemic areas are located in the states of Maranhão, Pará, Rio Grande do Norte, Paraíba, Pernambuco, Alagoas, Sergipe, Bahia, Minas Gerais, Espírito Santo and in the city of Rio de Janeiro. In Minas Gerais, the endemic areas are located in the mesoregions of Norte de Minas, Campo das Vertentes, Oeste de Minas, Jequitinhonha, Vale do Mucuri, Vale do Rio Doce, Metropolitana de Belo Horizonte, Zona da Mata and Central Mineira.

Among neotropical Biomphalaria, ten species and one subspecies are found in Brazil. Some species are widely distributed while others are restricted to some specific regions. The intermediate hosts of Schistosoma mansoni in Brazil are Biomphalaria glabrata (Say, 1818), Biomphalaria

\footnotetext{
${ }^{+}$Corresponding author. Fax: +55-31-3295.3115

E-mail: souzacp@cpqrr.fiocruz.br

Received 11 July 2000

Accepted 14 November 2000
}

tenagophila (Orbigny, 1835) and Biomphalaria straminea (Dunker, 1848). B. glabrata is epidemiologically the most important host regarding its wide geographic distribution and high susceptibility to infection by the trematode (Souza et al. 1995). Furthermore, its distribution is nearly always related to the presence of schistosomiasis. Two other species, Biomphalaria amazonica Paraense, 1966 and Biomphalaria peregrina (Orbigny, 1835) can be infected with $S$. mansoni under experimental conditions (Corrêa \& Paraense 1971, Paraense 1973).

Malacological surveys and isolated captures, undertaken by several researchers, have already recorded the presence of seven Biomphalaria species in Minas Gerais. The three snail hosts of $S$. mansoni occur in some municipalities, having been found naturally infected with $S$. mansoni and other trematode larvae, which do not infect man (Ruiz 1952, Souza et al. 1998b).

The present work was aimed to update the knowledge of the distribution of Biomphalaria species and to contribute to the elaboration of the planorbid chart of Minas Gerais.

\section{MATERIALS AND METHODS}

The list of municipalities of Minas Gerais where Biomphalaria (=Australorbis; Tropicorbis; Platytaphius; Taphius, Paraense 1958) occurs was organized with data of the literature and unpub- 
lished observations. The list is presented by mesoregions and municipalities (IGA 1995). Snails were collected in breeding places from different municipalities in Minas Gerais at different periods, using scoops and tweezers, and then packed to be transported to the laboratory (Souza \& Lima 1990). Specific identification was performed according to the morphology of the shells, reproductive system and renal ridge of the snails (Deslandes 1951, Paraense \& Deslandes 1955a,b, 1959, Paraense 1975, 1981), or recently through low stringency polymerase chain reaction and restriction fragment lenght polymorphism (Vidigal et al. 2000). Different researchers, whose works are quoted in the references, collected and identified the snails. In the last 40 years, great part of identification was undertaken by Dr Wladimir Lobato Paraense, Departamento de Malacologia, Instituto Oswaldo Cruz, Rio de Janeiro. In the last two decades technicians and researchers, mainly from Centro de Pesquisas René Rachou, Fiocruz, Belo Horizonte, Fundação Nacional de Saúde, Belo Horizonte and Universidade Federal de Minas Gerais, Belo Horizonte, have done their utmost to collect and classify the planorbid snails from this state (Souza et al. 1981a, 1998b, Souza 1986, Souza \& Lima 1997, Carvalho et al. 1989, 1994,
1997, 1998a, FNS 1995, 1998). A great deal of information was also obtained from materials sent by health officials of municipalities in the state, urban and rural state owners, researchers and technicians from several institutions.

The malacological survey was performed in all municipalities belonging to mesoregions of Triângulo Mineiro/Alto Paranaíba and Norte de Minas (Carvalho et al. 1994, 1997, 1998a). The municipalities in which the presence of any Biomphalaria species was noticed are recorded in the Table.

\section{RESULTS}

The occurrence of seven Biomphalaria species was recorded in Minas Gerais. The current distribution, per municipalities, in the twelve mesoregions in this state is shown in the Table and in the Figure. Snails were found in $283(33.1 \%)$ municipalities, out of 853 , with the following distribution: $B$. glabrata $65.3 \%$ (185) municipalities, $B$. straminea $43.8 \%$ (125), B. tenagophila $20.4 \%$ (58), $B$. peregrina $20.1 \%$ (57), B. schrammi $9.2 \%$ (26), $B$. intermedia $7 \%$ (20) and B. occidentalis $0.7 \%$ (2). The three snail hosts of $S$. mansoni in Brazil ( $B$. glabrata, B. straminea and B. tenagophila) were found in $24(8.4 \%)$ municipalities.

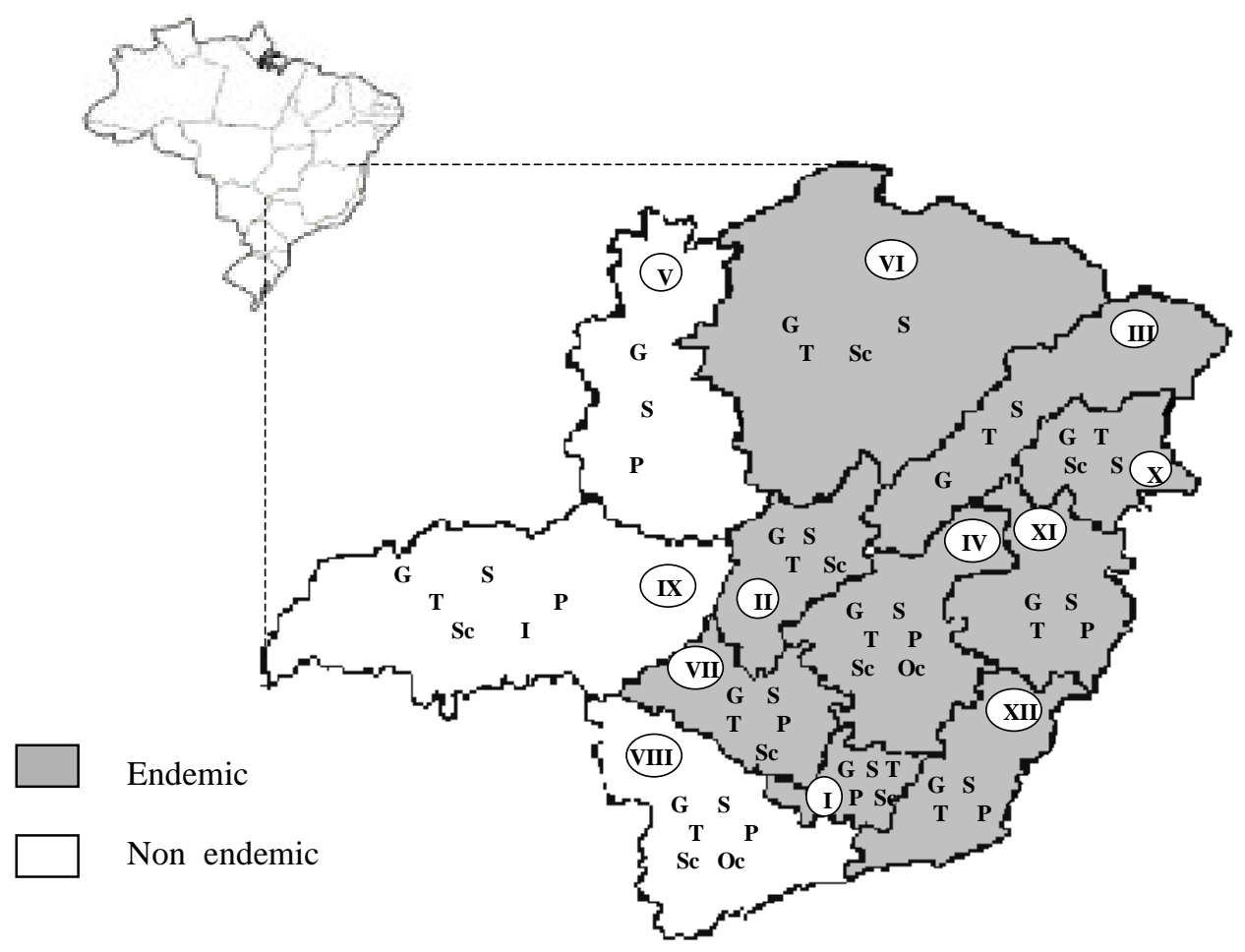

Map of Minas Gerais showing the endemic and non endemic mesoregions for schistosomiasis and the species of Biomphalaria. Mesoregions - I: Campo das Vertentes; II: Central Mineira; III: Jequitinhonha; IV: Metropolitana de Belo Horizonte; V: Noroeste de Minas; VI: Norte de Minas; VII: Oeste de Minas; VIII: Sul/Sudoeste de Minas; IX: Triângulo Mineiro/Alto Paranaíba; X: Vale do Mucuri; XI: Vale do Rio Doce; XII: Zona da Mata. G: B. glabrata; T: B. tenagophila; S: B. straminea; P: B. peregrina; Sc: B. schrammi; I: B. intermedia; Oc: B. occidentalis 
TABLE

Distribution of Biomphalaria snails per municipality in twelve mesoregions in the State of Minas Gerais

\begin{tabular}{|c|c|c|c|c|c|c|c|c|}
\hline \multirow{2}{*}{$\begin{array}{l}\text { Mesoregion } \\
\text { municipality }\end{array}$} & \multirow{2}{*}{$\begin{array}{l}\text { Geographic } \\
\text { coordinates }\end{array}$} & \multicolumn{7}{|c|}{ Biomphalaria species } \\
\hline & & $\mathrm{G}$ & $\mathrm{T}$ & $\mathrm{S}$ & $\mathrm{P}$ & $\mathrm{Sc}$ & I & Oc \\
\hline \multicolumn{9}{|l|}{ I Campo das Vertentes } \\
\hline 1) Barbacena & $21 \mathrm{~s} 13 / 43 w 46$ & 4 & 2 & - & 12 & - & - & - \\
\hline Caranaíba & $20 \mathrm{~s} 52 / 43 w 44$ & 11 & - & 11 & - & - & - & - \\
\hline Lavras & $21 \mathrm{~s} 14 / 45 \mathrm{w} 00$ & - & - & - & 5 & 54 & - & - \\
\hline Nepomuceno & $21 \mathrm{~s} 13 / 45 \mathrm{w} 14$ & - & - & - & - & 54 & - & - \\
\hline 5) São João del Rey & $21 \mathrm{~s} 08 / 44 \mathrm{w} 15$ & 4 & - & - & - & - & - & - \\
\hline \multicolumn{9}{|l|}{ II Central Mineira } \\
\hline 6) Bom Despacho & $19 \mathrm{~s} 44 / 45 w 15$ & - & 4 & - & - & - & - & - \\
\hline Buenópolis & $17 \mathrm{~s} 52 / 44 \mathrm{w} 01$ & 4 & - & 2 & - & - & - & - \\
\hline Corinto & $18 s 21 / 44 w 27$ & 4 & - & 2,7 & - & - & - & - \\
\hline 9) Curvelo & $18 \mathrm{~s} 45 / 44 w 25$ & 2,7 & - & 2,7 & - & - & - & - \\
\hline 10) Delta & $19 \mathrm{~s} 58 / 47 \mathrm{w} 46$ & - & 52 & 52 & - & - & - & - \\
\hline 11) Dores do Indaiá & $19 s 27 / 45 w 36$ & 2,7 & - & - & - & - & - & - \\
\hline 12) Inimutaba & $18 s 43 / 46 w 44$ & 52 & - & 52 & - & - & - & - \\
\hline 13) Lagoa da Prata & $20 \mathrm{~s} 01 / 45 \mathrm{w} 32$ & 4 & 8 & 4 & - & 4 & - & - \\
\hline 14) Trềs Marias & $18 \mathrm{~s} 12 / 45 w 15$ & - & - & 4 & - & - & - & - \\
\hline
\end{tabular}

III Jequitinhonha

15) Almenara

16s11/40w41

16) Caraí

17) Chapada do Norte

17s11/41w41

$17 \mathrm{~s}-5 / 42 \mathrm{w} 32$

$16 \mathrm{~s} 17 / 41 \mathrm{w} 47$

18 s $14 / 43$ w36

19) Diamantina

20) Itinga

21) Jacinto

22) Jequitinhonha

23) Joaima

24) Medina

25) Minas Nova

26) Novo Cruzeiro

$16 \mathrm{~s} 36 / 41 \mathrm{w} 46$

$16 \mathrm{~s} 08 / 40 \mathrm{w} 17$

16s26/41w00

16s39/41w01

16s $13 / 41 w 28$

$17 \mathrm{~s} 12 / 42 \mathrm{w} 35$

$17 \mathrm{~s} 28 / 41 \mathrm{w} 52$

16s00/41w16

20s14/42w53

28) Rio Doce

16s00/39w56

$\begin{array}{ccccccc}2,7 & - & - & - & - & - & - \\ 11 & 11 & - & - & - & - & - \\ - & - & 46 & - & - & - & - \\ 2,7 & - & - & - & - & - & - \\ 2,7 & - & - & - & - & - & - \\ 4 & - & 2,7 & - & - & - & - \\ 4 & - & 2,7 & - & - & - & - \\ 13 & - & - & - & - & - & - \\ 4 & 4 & - & - & - & - & - \\ 29 & - & - & - & - & - & - \\ - & - & 17 & - & - & - & - \\ 2,7 & - & - & - & - & - & - \\ 32 & - & - & - & - & - & - \\ 52 & 52 & - & - & - & - & - \\ 4 & - & 2,7 & - & - & - & -\end{array}$

IV Metropolitana de Belo Horizonte

30) Alvinópolis

20s06/43w02

31) Araçaí

32) Baldim

$19 \mathrm{~s} 12 / 44 \mathrm{w} 14$

19s $17 / 43$ w5 57

33) Barão de Cocais

$19 \mathrm{~s} 56 / 43 \mathrm{w} 29$

34) Belo Horizonte

19s55/43w56

35) Belo Vale

36) Betim

37) Brumadinho

38) Caeté

39) Capim Branco

40) Catas Altas da Noruega

20s24/44w01

$19 \mathrm{~s} 58 / 44 \mathrm{w} 11$

20s08/44w12

$19 \mathrm{~s} 53 / 43 \mathrm{w} 40$

19s32/44w07

20s41/43w29

41) Conceição do Mato Dentro

$19 \mathrm{~s} 02 / 43 \mathrm{w} 25$

42) Conselheiro Lafaiete

43) Contagem

20s39/43w47

19s56/44w02

19s07/44w19

$19 \mathrm{~s} 05 / 42 \mathrm{w} 46$

45) Dionísio

46) Dom Joaquim

$18 \mathrm{~s} 58 / 43 \mathrm{w} 15$

$19 \mathrm{~s} 45 / 44 \mathrm{w} 18$

19s13/43w01

$19 \mathrm{~s} 53 / 44 \mathrm{w} 25$

20s01/44w03

20s04/44w18

19 s $37 / 43$ w 13

$20 \mathrm{~s} 15 / 43 \mathrm{w} 48$

$19 \mathrm{~s} 31 / 43 \mathrm{w} 44$

19s $14 / 44 w 01$

54) Jaboticatubas

55) Jequitibá

19s48/43w01 


\begin{tabular}{|c|c|c|c|c|c|c|c|c|}
\hline \multirow{2}{*}{$\begin{array}{l}\text { Mesoregion } \\
\text { municipality }\end{array}$} & \multirow{2}{*}{$\begin{array}{l}\text { Geographic } \\
\text { coordinates }\end{array}$} & \multicolumn{7}{|c|}{ Biomphalaria species } \\
\hline & & $\mathrm{G}$ & $\mathrm{T}$ & $\mathrm{S}$ & $\mathrm{P}$ & Sc & I & Oc \\
\hline 57) Lagoa Santa & $19 s 37 / 45 w 53$ & 24 & 4 & 7,48 & - & 42 & - & - \\
\hline 58) Mariana & $20 \mathrm{~s} 22 / 43 \mathrm{w} 24$ & 45 & - & - & - & - & - & - \\
\hline 59) Mateus Leme & $19 \mathrm{~s} 59 / 44 w 25$ & 8 & - & 17 & - & - & - & - \\
\hline 60) Matozinhos & 19s33/44w04 & 4 & - & 33 & - & - & - & - \\
\hline 61) Nova Era & $19 \mathrm{~s} 45 / 43 \mathrm{w} 02$ & 28 & 28 & - & - & - & - & - \\
\hline 62) Nova Lima & $19 \mathrm{~s} 59 / 43 w 05$ & 2,7 & 4 & 4 & 5 & - & - & - \\
\hline 63) Nova União & $19 s 41 / 43 w 34$ & 8 & - & - & - & - & - & - \\
\hline 64) Ouro Branco & $20 \mathrm{~s} 31 / 43 w 41$ & 31 & 31 & - & - & - & - & - \\
\hline 65) Ouro Preto & $20 \mathrm{~s} 23 / 43 w 03$ & 28 & - & - & - & - & - & - \\
\hline 66) Papagaios & $19 s 26 / 44 w 44$ & 4 & - & 46 & - & 4 & - & - \\
\hline 67) Paraopeba & $19 \mathrm{~s} 16 / 44 \mathrm{w} 24$ & 52 & - & 40 & - & - & - & - \\
\hline 68) Pará de Minas & $19 s 51 / 44 w 36$ & 40 & - & - & - & - & - & - \\
\hline 69) Pedro Leopoldo & 19 s37/44w02 & 33 & - & $2,7,48$ & 5 & - & - & - \\
\hline 70) Pitangui & $19 s 40 / 56 w 44$ & 52 & 52 & 52 & - & - & - & - \\
\hline 71) Prudente de Morais & 19 s28/44w09 & - & - & 40 & - & - & - & - \\
\hline 72) Ribeirão das Neves & $19 \mathrm{~s} 45 / 44 w 05$ & 33 & - & - & - & - & - & - \\
\hline 73) Rio Acima & $20 \mathrm{~s} 05 / 43 w 47$ & 43 & - & 43 & - & - & - & - \\
\hline 74) Rio Piracicaba & $19 \mathrm{~s} 55 / 43 w 01$ & 2,7 & - & - & - & - & - & - \\
\hline 75) Rio Vermelho & $18 \mathrm{~s} 17 / 43 \mathrm{w} 00$ & 2 & - & - & - & - & - & - \\
\hline 76) Sabará & $18 s 53 / 43 w 48$ & 28 & 4 & 4 & 4 & - & - & - \\
\hline 77) Santa Bárbara & $19 \mathrm{~s} 57 / 43 w 24$ & 28 & - & - & - & - & - & - \\
\hline 78) Santa Luzia & $19 \mathrm{~s} 46 / 43 \mathrm{w} 51$ & 35 & 8 & 8 & - & - & - & - \\
\hline 79) Santa Maria de Itabira & $19 \mathrm{~s} 27 / 43 \mathrm{w} 06$ & 36 & - & - & - & - & - & - \\
\hline 80) Santana do Pirapama & $19 \mathrm{~s} 00 / 44 \mathrm{w} 02$ & - & - & 27 & - & - & - & - \\
\hline 81) Santana do Riacho & $19 \mathrm{~s} 01 / 43 \mathrm{w} 42$ & 42 & - & - & - & - & - & - \\
\hline 82) São Sebastião do Rio Preto & 19s17/43w01 & 2,7 & - & - & - & - & - & - \\
\hline 83) Sete Lagoas & 19 s27/44w14 & 2,7 & 4 & 2,7 & - & - & - & - \\
\hline 84) Taquaraçu de Minas & $19 \mathrm{~s} 04 / 43 \mathrm{w} 41$ & 4 & 4 & 4 & - & - & - & - \\
\hline 85) Vespasiano & $19 \mathrm{~s} 41 / 43 w 55$ & 2,7 & 4 & 28 & - & - & - & - \\
\hline \multicolumn{9}{|l|}{ V Noroeste de Minas } \\
\hline 86) Bonfinópolis de Minas & $16 \mathrm{~s} 34 / 45 w 59$ & - & - & 16 & - & - & - & - \\
\hline 87) João Pinheiro & $17 \mathrm{~s} 44 / 46 w 01$ & - & - & 16 & - & - & - & - \\
\hline 88) Lagamar & $18 \mathrm{~s} 01 / 46 w 48$ & - & - & 16 & - & - & - & - \\
\hline 89) Lagoa Grande & $17 \mathrm{~s} 05 / 46 \mathrm{w} 31$ & - & - & 16 & - & - & - & - \\
\hline 90) Paracatu & $17 \mathrm{~s} 13 / 46 w 52$ & 4 & - & 2,7 & - & - & - & - \\
\hline 91) Presidente Olegário & $18 \mathrm{~s} 24 / 46 w 25$ & - & - & - & 16 & - & - & - \\
\hline 92) São Gonçalo do Abaeté & $18 \mathrm{~s} 20 / 18 w 45$ & 52 & - & 52 & - & - & - & - \\
\hline 93) Unaí & $16 \mathrm{~s} 21 / 46 w 54$ & - & - & 16 & - & - & - & - \\
\hline 94) Vazante & $17 \mathrm{~s} 59 / 46 w 54$ & - & - & 16 & - & - & - & - \\
\hline
\end{tabular}

VI Norte de Minas

95) Águas Vermelhas

96) Bocaiúva

97) Brasília de Minas

98) Capitão Enéas

99) Claro dos Poções

100) Coração de Jesus

101) Engenheiro Navarro

102) Espinosa

103) Francisco Sá

104) Ibiaí

105) Ibiracatu

106) Itacarambí

107) Janaúba

108) Januária

109) Japonvar

110) Jequitaí

111) Juramento

112) Lagoa dos Patos

113) Lassance

114) Lontra

115) Mamonas

116) Manga

117) Matias Barbosa

$15 s 44 / 41 w 27$ $17 \mathrm{~s} 06 / 43 \mathrm{w} 48$ $16 \mathrm{~s} 12 / 44 \mathrm{w} 25$ $16 \mathrm{~s} 19 / 43 \mathrm{w} 42$ $17 \mathrm{~s}-4 / 44 \mathrm{w} 12$ $16 \mathrm{~s} 41 / 44 \mathrm{w} 21$ 17s16/43w57 $19 \mathrm{~s} 55 / 42 \mathrm{w} 48$ 16 s $28 / 43$ w 29

16s51/44w54

19s39/44w10

15s06/44w05

$15 \mathrm{~s} 48 / 43 \mathrm{w} 18$

15s29/44w 21

15s59/44w16

17s $13 / 44 w 26$

$16 \mathrm{~s} 05 / 43 \mathrm{w} 35$

16s58/44w34

17s53/44w34

$15 \mathrm{~s} 54 / 44 \mathrm{w} 18$

$15 \mathrm{~s}-3 / 42 \mathrm{w} 56$

$14 \mathrm{~s} 45 / 43 \mathrm{w} 55$

$21 \mathrm{~s} 52 / 09 \mathrm{w} 43$

$\begin{array}{ccccccc}4 & - & - & - & - & - & - \\ 2,7 & 51 & 2,7 & - & - & - & - \\ 2,7 & - & 51 & - & - & - & - \\ - & - & 18 & - & - & - & - \\ 18 & - & 18 & - & - & - & - \\ 2,7 & - & 51 & - & - & - & - \\ 18 & - & 18 & - & - & - & - \\ 4 & - & 2 & - & - & - & - \\ 2,7 & - & 25 & - & - & - & - \\ 18 & - & 18 & - & - & - & - \\ 52 & - & 52 & - & - & - & - \\ 4 & - & 2 & - & - & - & - \\ 2 & - & 51 & - & - & - & - \\ 26 & - & 51 & - & - & - & - \\ 52 & - & 52 & - & - & - & - \\ 7 & 7 & 2 & - & - & - & - \\ - & - & 27 & - & - & - & - \\ 18 & - & 18 & - & - & - & - \\ - & - & - & - & 32 & - & - \\ 4 & - & 2 & - & - & - & - \\ 4 & - & 51 & - & - & - & - \\ 4 & - & - & - & - & - & - \\ - & 52 & - & - & - & - & -\end{array}$




\begin{tabular}{|c|c|c|c|c|c|c|c|c|}
\hline \multirow{2}{*}{$\begin{array}{l}\text { Mesoregion } \\
\text { municipality }\end{array}$} & \multirow{2}{*}{$\begin{array}{l}\text { Geographic } \\
\text { coordinates }\end{array}$} & \multicolumn{7}{|c|}{ Biomphalaria species } \\
\hline & & G & $\mathrm{T}$ & $\mathrm{S}$ & $\mathrm{P}$ & $\mathrm{Sc}$ & I & $\mathrm{Oc}$ \\
\hline 118) Mato Verde & $15 \mathrm{~s} 23 / 42 \mathrm{w} 51$ & - & - & 51 & - & - & - & - \\
\hline 119) Mirabela & $16 s 15 / 44 w 09$ & 18 & - & 18 & - & - & - & - \\
\hline 120) Montalvânia & $14 \mathrm{~s} 25 / 44 w 21$ & 4 & - & 8 & - & - & - & - \\
\hline 121) Montes Claros & $16 s 44 / 43 w 51$ & 25 & - & 2 & - & - & - & - \\
\hline 122) Pirapora & $17 \mathrm{~s} 02 / 44 w 56$ & 4 & - & 2,7 & - & 55 & - & - \\
\hline 123) Porteirinha & $15 \mathrm{~s} 44 / 43 \mathrm{w} 01$ & 4 & - & 2,7 & - & - & - & - \\
\hline 124) Rio Pardo de Minas & $15 \mathrm{~s} 36 / 42 \mathrm{w} 32$ & 2,7 & - & - & - & - & - & - \\
\hline 125) Rubelita & $16 \mathrm{~s} 24 / 42 \mathrm{w} 15$ & 51 & - & - & - & - & - & - \\
\hline 126) Salinas & $16 \mathrm{~s} 01 / 42 \mathrm{w} 17$ & 32 & - & 25 & - & - & - & - \\
\hline 127) São João da Ponte & $15 \mathrm{~s} 55 / 44 \mathrm{w} 00$ & 18 & - & 7 & - & - & - & - \\
\hline 128) São João do Paraíso & $15 \mathrm{~s} 18 / 42 \mathrm{w} 00$ & 2,7 & - & - & - & - & - & - \\
\hline 129) São Romão & $16 \mathrm{~s} 22 / 45 w 04$ & 4 & - & 2,7 & - & - & - & - \\
\hline 130) Ubai & $16 s 17 / 44 w 46$ & 18 & 18 & 18 & - & - & - & - \\
\hline 131) Varzelândia & $15 \mathrm{~s} 42 / 44 \mathrm{w} 01$ & 18 & 8 & 18 & - & - & - & - \\
\hline \multicolumn{9}{|l|}{ VII Oeste de Minas } \\
\hline 132) Arcos & $20 \mathrm{~s} 16 / 45 w 32$ & 4 & - & 2,7 & - & - & - & - \\
\hline 133) Bambuí & $20 \mathrm{~s} 00 / 45 w 58$ & 10 & - & 8 & - & 33 & - & - \\
\hline 134) Divinópolis & $20 \mathrm{~s} 08 / 44 w 53$ & 4 & 4 & - & - & - & - & - \\
\hline 135) Doresópolis & $20 \mathrm{~s} 17 / 45 w 54$ & 4 & 4 & - & - & 4 & - & - \\
\hline 136) Formiga & $20 \mathrm{~s} 27 / 45 w 25$ & 32 & - & - & - & - & - & - \\
\hline 137) Itaúna & $20 s-4 / 44 w 34$ & 4 & - & 8 & - & - & - & - \\
\hline 138) Pains & $20 \mathrm{~s} 22 / 45 w 39$ & 2,7 & - & 52 & - & - & - & - \\
\hline 139) Pedra do Indaiá & $20 \mathrm{~s} 15 / 45 w 12$ & - & - & - & 5 & - & - & - \\
\hline \multicolumn{9}{|l|}{ VIII Sul/Sudoeste de Minas } \\
\hline 140) Alfenas & $21 \mathrm{~s} 25 / 45 w 56$ & - & 4 & - & 5 & 54 & - & - \\
\hline 141) Alpinópolis & $20 \mathrm{~s} 51 / 46 w 23$ & - & - & - & 6 & - & - & - \\
\hline 142) Alterosa & $21 \mathrm{~s} 14 / 46 w 08$ & 2,7 & - & - & 5 & - & - & - \\
\hline 143) Andradas & $22 \mathrm{~s} 04 / 46 w 34$ & 7 & - & - & - & - & - & - \\
\hline 144) Areado & 21s21/46w08 & - & - & - & 4 & - & - & - \\
\hline 145) Bom Jesus da Penha & $21 \mathrm{~s} 01 / 46 \mathrm{w} 31$ & - & - & - & 39 & - & - & - \\
\hline 146) Borda da Mata & $22 \mathrm{~s} 16 / 46 w 09$ & - & - & - & 12 & - & - & - \\
\hline 147) Bueno Brandão & $22 \mathrm{~s} 26 / 46 w 21$ & - & - & 7 & - & - & - & - \\
\hline 148) Cabo Verde & $21 \mathrm{~s} 28 / 46 w 23$ & - & 17 & - & 6 & - & - & - \\
\hline 149) Campanha & $21 \mathrm{~s} 05 / 45 w 24$ & 4 & - & - & - & - & - & - \\
\hline 150) Campo do Meio & $21 \mathrm{~s} 06 / 45 w 49$ & - & - & - & 5 & - & - & - \\
\hline 151) Campos Gerais & $21 \mathrm{~s} 14 / 45 \mathrm{w} 45$ & - & - & 8 & - & 54 & - & - \\
\hline 152) Capetinga & 20s37/47w03 & - & - & - & - & - & - & 39 \\
\hline 153) Capitólio & $20 \mathrm{~s} 36 / 46 w 03$ & - & - & - & 6 & - & - & - \\
\hline 154) Carmo do Rio Claro & $20 \mathrm{~s} 58 / 46 w 07$ & - & - & - & 5 & - & - & - \\
\hline 155) Cassia & $20 \mathrm{~s} 35 / 46 w 55$ & - & - & 6 & - & 39 & - & - \\
\hline 156) Claraval & $20 \mathrm{~s} 23 / 47 w 16$ & - & - & 6 & - & - & - & - \\
\hline 157) Conceição da Aparecida & $21 \mathrm{~s} 05 / 46 \mathrm{w} 12$ & 7 & - & - & 5 & - & - & - \\
\hline 158) Delfinópolis & $20 \mathrm{~s} 02 / 46 w 51$ & - & - & 6 & - & - & - & - \\
\hline 159) Eloi Mendes & $21 \mathrm{~s} 36 / 45 w 33$ & - & - & - & 5 & - & - & - \\
\hline 160) Fama & $21 \mathrm{~s} 24 / 45 w 49$ & - & - & - & 5 & - & - & - \\
\hline 161) Fortaleza de Minas & $20 \mathrm{~s} 50 / 46 w 42$ & 32 & - & - & - & - & - & - \\
\hline 162) Guaranésia & $21 \mathrm{~s} 17 / 46 \mathrm{w} 48$ & - & 6 & - & 6 & - & - & - \\
\hline 163) Guaxupé & $21 \mathrm{~s} 18 / 46 w 42$ & - & 6 & - & 6 & 6 & - & - \\
\hline 164) Itajubá & $22 \mathrm{~s} 25 / 45 w 27$ & 51 & 23 & - & 17 & 54 & - & - \\
\hline 165) Itamoji & $21 \mathrm{~s} 04 / 47 w 02$ & - & - & - & 6 & - & - & - \\
\hline 166) Itaú de Minas & $20 \mathrm{~s} 44 / 43 w 45$ & - & - & - & - & 6 & - & - \\
\hline 167) Jacuí & $21 \mathrm{~s} 01 / 46 \mathrm{w} 44$ & - & - & - & 6 & - & - & - \\
\hline 168) Juruaia & 21s15/46w34 & - & - & - & 5 & - & - & - \\
\hline 169) Machado & $21 \mathrm{~s} 39 / 45 w 55$ & - & - & - & 5 & - & - & - \\
\hline 170) Monte Belo & $21 \mathrm{~s} 19 / 46 w 22$ & - & 6 & - & 5 & - & - & - \\
\hline 171) Monte Santo de Minas & $21 \mathrm{~s} 11 / 46 w 58$ & - & - & - & 6 & - & - & - \\
\hline 172) Muzambinho & $21 \mathrm{~s} 22 / 46 w 31$ & - & - & - & 6 & - & - & - \\
\hline 173) Ouro Fino & $22 \mathrm{~s} 16 / 46 w 22$ & - & - & - & 12 & 54 & - & - \\
\hline 174) Paraguaçu & $21 \mathrm{~s} 32 / 45 w 44$ & - & 52 & 52 & 5 & - & - & - \\
\hline 175) Paraisópolis & $22 \mathrm{~s} 33 / 45 w 46$ & - & - & - & 4 & 4 & - & - \\
\hline 176) Passos & $20 s 43 / 46 w 36$ & 8 & 8 & 8 & 12 & - & - & - \\
\hline 177) Pouso Alegre & $22 \mathrm{~s} 13 / 45 w 56$ & - & - & - & 12 & 53 & - & - \\
\hline 178) Santa Rita do Sapucaí & $22 \mathrm{~s} 15 / 45 \mathrm{w} 42$ & - & - & - & 12 & - & - & - \\
\hline 179) Santana da Vargem & $21 \mathrm{~s} 15 / 45 \mathrm{w} 03$ & - & - & - & 5 & - & - & $-i$ \\
\hline
\end{tabular}




\begin{tabular}{|c|c|c|c|c|c|c|c|c|}
\hline \multirow{2}{*}{$\begin{array}{l}\text { Mesoregion } \\
\text { municipality }\end{array}$} & \multirow{2}{*}{$\begin{array}{l}\text { Geographic } \\
\text { coordinates }\end{array}$} & \multicolumn{7}{|c|}{ Biomphalaria species } \\
\hline & & $\mathrm{G}$ & $\mathrm{T}$ & $\mathrm{S}$ & $\mathrm{P}$ & $\mathrm{Sc}$ & I & Oc \\
\hline 180) São João Batista do Gloria & $20 \mathrm{~s} 38 / 46 w 03$ & - & - & - & 6 & - & - & - \\
\hline 181) São Pedro da União & $21 \mathrm{~s} 07 / 46 w 36$ & - & - & - & 6 & - & - & - \\
\hline 182) São Sebastião do Paraíso & $20 \mathrm{~s} 55 / 46 w 59$ & - & - & 6 & - & - & - & - \\
\hline 183) São Tomaz de Aquino & $20 \mathrm{~s} 47 / 47 w 05$ & - & - & 6 & - & - & - & - \\
\hline 184) Sapacuí Mirim & $22 \mathrm{~s} 44 / 45 w 44$ & 51 & - & - & - & - & - & - \\
\hline 185) Silvianópolis & $22 \mathrm{~s} 01 / 45 \mathrm{w} 05$ & - & - & - & 5 & - & - & - \\
\hline 186) Três Corações & $21 \mathrm{~s} 41 / 45 \mathrm{w} 15$ & - & - & - & 5 & - & - & - \\
\hline 187) Três Pontas & $21 \mathrm{~s} 22 / 45 \mathrm{w} 03$ & - & - & - & 5 & 54 & - & - \\
\hline 188) Turvolândia & $21 \mathrm{~s} 52 / 45 \mathrm{w} 47$ & - & 7 & - & - & - & - & - \\
\hline
\end{tabular}

IX Triângulo Mineiro/Alto Paranaíba

189) Abadia dos Dourados 18s29/47w24

190) Água Comprida 20s03/48w06

191) Araxá 19s35/46w56

192) Cachoeira Dourada 18s03/49w03

193) Campina Verde 19s32/49w29

194) Canápolis $18 \mathrm{~s} 43 / 49 \mathrm{w} 12$

195) Capinópolis 18s04/49w34

196) Cascalho Rico 18s34/47w52

197) Centralina 18s35/49w11

198) Conceição das Alagoas $19 s 54 / 48 w 23$

199) Coromandel 18s $28 / 47 \mathrm{w} 12$

200) Douradoquara 18s25/47w36

201) Fronteira 20s $17 / 49 w 12$

202) Grupiara

203) Guimarânia

$18 \mathrm{~s} 29 / 47 \mathrm{w} 43$

$18 \mathrm{~s} 05 / 46 \mathrm{w} 47$

$19 \mathrm{~s} 12 / 49 \mathrm{w} 47$

$18 \mathrm{~s} 41 / 49 \mathrm{w} 56$

205) Ipiaçu

206) Itapajipe

207) Itambacurí

$19 \mathrm{~s} 54 / 49 \mathrm{w} 21$

$18 \mathrm{~s} 01 / 41 \mathrm{w} 41$

$18 \mathrm{~s} 58 / 49 \mathrm{w} 27$

208) Ituiutaba

$19 \mathrm{~s} 43 / 05 \mathrm{w} 11$

$18 \mathrm{~s} 46 / 46 \mathrm{w} 24$

$18 \mathrm{~s} 52 / 48 \mathrm{w} 52$

$18 \mathrm{~s} 43 / 47 \mathrm{w} 29$

$18 \mathrm{~s} 35 / 46 w 31$

212) Monte Carmelo

213) Patos de Minas

214) Patrocínio

215) Planura

216) Sacramento

217) Santa Vitória

218) São Francisco Sales

219) Tapira

220) Tupaciguara

221) Uberaba

222) Uberlândia

18s56/46w59

20s08/48w42

$19 \mathrm{~s} 51 / 47 \mathrm{w} 26$

18s05/05w07

19s51/49w46

$19 \mathrm{~s} 55 / 46 w 49$

$18 \mathrm{~s} 35 / 48 \mathrm{w} 42$

19s45/47w56

18 s55/48w16

X Vale do Mucuri

223) Malacacheta

$17 \mathrm{~s} 05 / 42 \mathrm{w} 04$

$17 \mathrm{~s} 04 / 04 \mathrm{w} 43$

$17 \mathrm{~s} 05 / 04 \mathrm{w} 21$

225) Nanuque

226) Teófilo Otoni

17s51/41w03

$\begin{array}{ccccccc}- & - & - & 1 & - & - & - \\ - & 3 & - & - & - & 3 & - \\ - & - & 52 & - & - & 52 & - \\ - & - & 3 & - & - & - & - \\ - & - & - & - & - & 3 & - \\ - & - & - & - & - & 3 & - \\ - & - & - & - & - & 3 & - \\ - & - & 3 & - & - & - & - \\ - & - & 3 & - & - & 3 & - \\ - & - & 3 & - & - & 3 & - \\ - & - & - & 1 & - & - & - \\ - & - & 1 & - & - & - & - \\ - & - & 1 & - & - & 3 & - \\ - & - & - & 1 & - & - & - \\ - & - & - & - & - & 3 & - \\ - & - & 3 & - & - & 3 & - \\ - & - & - & - & - & 3 & - \\ 19 & - & - & - & - & - & - \\ - & - & 3 & - & - & 3 & - \\ - & - & - & - & - & 3 & - \\ - & - & - & - & 1 & - & - \\ 8 & - & 3 & - & - & 3 & - \\ - & - & - & - & - & 1 & - \\ - & 1 & - & - & - & - & - \\ - & - & - & 1 & - & - & - \\ - & - & - & - & - & 3 & - \\ 1 & - & 1 & - & - & 1 & - \\ - & - & 3 & - & - & - & - \\ - & - & - & - & - & 3 & - \\ 8 & - & - & 1 & - & 1 & - \\ - & - & - & - & - & 3 & - \\ - & 3 & 3 & 5 & - & - & - \\ 52 & 4 & 3 & 5 & - & - & -\end{array}$

XI Vale do Rio Doce

227) Água Boa

17s59/42w23

228) Aimorés

229) Antônio Dias

19s29/41w03

19s39/42w52

19s $13 / 42$ w 29

19s49/42w 18

231) Bom Jesus do Galho

$19 \mathrm{~s} 03 / 42 \mathrm{w} 42$

$18 \mathrm{~s} 14 / 41 \mathrm{w} 43$

$19 \mathrm{~s} 47 / 42 \mathrm{w} 08$

$18 \mathrm{~s} 14 / 42 \mathrm{w} 05$

19s01/41w28

19 s $31 / 42$ w 37

18 s48/42w36

19s12/42w02 


\begin{tabular}{|c|c|c|c|c|c|c|c|c|}
\hline \multirow{2}{*}{$\begin{array}{l}\text { Mesoregion } \\
\text { municipality }\end{array}$} & \multirow{2}{*}{$\begin{array}{l}\text { Geographic } \\
\text { coordinates }\end{array}$} & \multicolumn{7}{|c|}{ Biomphalaria species } \\
\hline & & $\mathrm{G}$ & $\mathrm{T}$ & $\mathrm{S}$ & $\mathrm{P}$ & $\mathrm{Sc}$ & I & Oc \\
\hline 240) Entre Folhas & 19 s37/42w13 & 2 & - & - & - & - & - & - \\
\hline 241) Frei Inocêncio & $18 \mathrm{~s} 33 / 41 w 54$ & 4 & - & 4 & - & - & - & - \\
\hline 242) Galiléia & $19 \mathrm{~s} 00 / 41 w 32$ & 2,7 & - & - & - & - & - & - \\
\hline 243) Governador Valadares & $18 \mathrm{~s} 51 / 41 w 56$ & 57 & 8 & 22 & - & 12 & - & - \\
\hline 244) Guanhães & $18 \mathrm{~s} 46 / 42 w 55$ & 2,7 & - & - & - & - & - & - \\
\hline 245) Iapu & $19 \mathrm{~s} 26 / 42 \mathrm{w} 13$ & 36 & - & - & - & - & - & - \\
\hline 246) Inhapim & $19 \mathrm{~s} 32 / 42 \mathrm{w} 07$ & 36 & - & - & - & - & - & - \\
\hline 247) Itabirinha de Mantena & $18 \mathrm{~s} 33 / 31$ w 13 & 4 & - & 4 & 4 & - & - & - \\
\hline 248) Itanhomi & $19 \mathrm{~s} 01 / 41 w 51$ & 36 & - & - & - & - & - & - \\
\hline 249) Itueta & $18 \mathrm{~s} 58 / 49 w 27$ & 38 & - & - & - & - & - & - \\
\hline 250) Jaguaraçu & $19 \mathrm{~s} 38 / 42 \mathrm{w} 45$ & 41 & 41 & - & - & - & - & - \\
\hline 251) Jampruca & $18 \mathrm{~s} 27 / 41 w 48$ & 2 & - & - & - & - & - & - \\
\hline 252) Mantena & $18 s 46 / 04 w 58$ & 21 & - & - & - & - & - & - \\
\hline 253) Marliéria & $19 s 42 / 42 w 43$ & 50 & 50 & - & - & - & - & - \\
\hline 254) Mesquita & $18 \mathrm{~s} 13 / 42 \mathrm{w} 36$ & 36 & - & - & - & - & - & - \\
\hline 255) Mutum & $19 s 49 / 41 w 26$ & 36 & 8 & 51 & 4 & - & - & - \\
\hline 256) Nacip Raydan & $18 \mathrm{~s} 27 / 42 \mathrm{w} 14$ & 4 & - & - & - & - & - & - \\
\hline 257) Peçanha & $18 \mathrm{~s} 32 / 42 w 33$ & 36 & - & - & - & - & - & - \\
\hline 258) Pocrane & 19 s $37 / 41 w 38$ & 7 & - & - & - & - & - & - \\
\hline 259) Resplendor & $19 \mathrm{~s} 19 / 41 w 15$ & 38 & - & - & - & - & - & - \\
\hline 260) Sabinópolis & $18 \mathrm{~s} 39 / 43 w 04$ & 2,7 & - & - & - & - & - & - \\
\hline 261) Santa Maria do Suaçuí & $18 \mathrm{~s} 11 / 42 \mathrm{w} 24$ & 2,7 & - & 4 & - & 54 & - & - \\
\hline 262) São João Evangelista & $18 \mathrm{~s} 32 / 42 \mathrm{w} 45$ & 36 & - & - & - & - & - & - \\
\hline 263) São Pedro do Suaçuí & $18 \mathrm{~s} 21 / 42 \mathrm{w} 36$ & 2,7 & - & 2,7 & - & - & - & - \\
\hline 264) Sobrália & 19s14/42w05 & - & - & 4 & - & - & - & - \\
\hline 265) Tarumirim & $19 \mathrm{~s} 16 / 42 \mathrm{w} 00$ & 13 & - & - & - & - & - & - \\
\hline 266) Timóteo & $19 \mathrm{~s} 34 / 42 w 38$ & 7 & - & 51 & - & - & - & - \\
\hline 267) Tumiritinga & $18 \mathrm{~s} 58 / 41 \mathrm{w} 38$ & 38 & - & - & - & - & - & - \\
\hline 268) Virgolândia & $18 \mathrm{~s} 28 / 42 \mathrm{w} 18$ & 8 & - & - & - & - & - & - \\
\hline \multicolumn{9}{|l|}{ XII Zona da Mata } \\
\hline 269) Barão de Monte Alto & $21 \mathrm{~s} 14 / 42 \mathrm{w} 14$ & - & 8 & - & - & - & - & - \\
\hline 270) Divino & 20 s36/42w08 & 33 & - & - & - & - & - & - \\
\hline 271) Juiz de Fora & $21 \mathrm{~s} 45 / 43 w 21$ & 28 & 28 & - & 12 & - & - & - \\
\hline 272) Manhuaçu & $20 \mathrm{~s} 15 / 42 \mathrm{w} 02$ & 2,7 & - & - & - & - & - & - \\
\hline 273) Matipó & $20 \mathrm{~s} 17 / 42 \mathrm{w} 02$ & 8 & - & - & - & - & - & - \\
\hline 274) Santo Antônio do Grama & $20 \mathrm{~s} 18 / 42 \mathrm{w} 36$ & 2,7 & - & - & - & - & - & - \\
\hline 275) Piau & $21 \mathrm{~s} 03 / 43 \mathrm{w} 19$ & 2,7 & - & - & - & - & - & - \\
\hline 276) Piedade de Ponte Nova & 20 s14/42w44 & 8 & - & - & - & - & - & - \\
\hline 277) Pirapetinga & 21s39/42w02 & 40 & - & - & - & - & - & - \\
\hline 278) Ponte Nova & 20 s24/42w54 & 2,7 & 52 & 57 & - & - & - & - \\
\hline 279) Rio Casca & 20 s $13 / 42$ w39 & 4 & - & 58 & - & - & - & - \\
\hline 280) Rio Novo & 21s28/43w07 & 4 & 2,7 & - & - & - & - & - \\
\hline 281) Tabuleiro & $21 \mathrm{~s} 21 / 43 \mathrm{w} 14$ & 2,7 & - & - & - & - & - & - \\
\hline 282) Ubá & $21 \mathrm{~s} 12 / 42 \mathrm{w} 53$ & 2,7 & 2 & 7 & - & - & - & - \\
\hline 283) Viçosa & $20 \mathrm{~s} 45 / 42 \mathrm{w} 52$ & 2,7 & 52 & - & - & - & - & - \\
\hline Total & - & 185 & 57 & 125 & 58 & 26 & 20 & 2 \\
\hline
\end{tabular}

G: B. glabrata; T: B. tenagophila; S: B. straminea; P: B. peregrina; Sc: B. schrammi; I: B. intermedia; Oc: B. occidentalis

The numbers in the columns below Biomphalaria species in the Table refer to the authors who recorded the occurrence of each species for the first time. 1: Carvalho et al. (1997); 2: Paraense (1972); 3: Carvalho et al. (1994); 4: Souza \& Lima (1990, 1997); 5: Paraense (1966); 6: Carvalho et al. (unpublished); 7: Freitas (1972); 8: Souza (unpublished); 9: Melo \& Pereira (1985); 10: Dias (1953); 11: Souza \& Lambertucci (unpublished); 12: Paraense \& Deslandes (1956a); 13: Paraense \& Deslandes (1959); 14: Teixeira (1919); 15: Paraense \& Deslandes (1956b); 16: Carvalho et al. (1998); 17: Souza (1986); 18: FNS (1995); 19: Pinto (1944); 20: Lima et al. (1993); 21: Brener \& Mourão (1956); 22: Penido et al. (1951); 23: Paraense \& Deslandes (1955b); 24: Paraense \& Santos (1953); 25: Martins \& Versiani (1938); 26: Baker (1945); 27: Souza et al. (1981); 28: Pinto \& Deslandes (1953); 29: Barbosa \& Dobbin (1951); 30: Paraense et al. (1954); 31: Silva et al. (1994); 32: Martins (1938); 33: Lucena (1956); 34: Martins et al. (1953); 35: Paraense (1955); 36: Andrade (1956); 37: Pinto \& Almeida (1945a); 38: Basseres \& Pantoja (1947); 39: Vidigal et al. (2000) ; 40: Souza \& Guimarães (unpublished); 41: Freitas (1978); 42: Souza et al. (1998a); 43: Souza et al. (1998b); 44: Barbosa et al. (1958); 45: Ruiz (1952); 46: Souza et al. (1983); 47: Ruiz (1951); 48: Freitas et al. (1972); 49: Souza et al. (1997); 50: Souza \& Coura-Filho (unpublished); 51: FNS (1998); 52: Melo (unpublished); 53: Pinto \& Almeida (1945b); 54: Paraense et al. (1964); 55: Oliveira \& Castro (1979); 56: Katz et al. (1970); 57: Pinto \& Almeida (1948); 58: Barros (1934) 


\section{DISCUSSION}

The need for updated information on geographic distribution of parasite vectors, in endemic regions, has led urban and rural communities up to seek institutions responsible for public health, aimed to know the transmission risks of diseases by those organisms. In the summer, mainly on vacation periods or prolonged holidays, the flow of people towards rural regions with lakes, rivers and waterfalls raises the dispersion risks of diseases caused by protozoa, helminths and viruses. In Brazil, schistosomiasis prevalence justifies the necessity of updated knowledge of the localization of intermediate hosts in risk regions (Souza et al. 1998a). Besides, the presence in a shorter scale of angiostrongyliasis and fascioliasis also play an important role, since these diseases are transmitted by snails.

Indeed, the real geographic distribution of schistosomiasis the transmitter snails in Brazil is not well known owing to the great territorial extension and the lack of human and economic resources (Paraense 1972). However, the number of studies aimed to better know the malacological fauna in some regions has increased in the last decades (Paraense 1983, 1986, Paraense et al. 1983, Souza 1986, Souza \& Lima 1997, Souza et al. 1998a,b, Teles 1987, 1989, 1996, Teles et al. 1991, Carvalho et al. 1989, 1994, 1997, 1998a,b, FNS 1995, 1998, Schlemper Junior et al. 1996).

In the present study the distribution of Biomphalaria snails per municipality and mesoregion in Minas Gerais (Table, Figure) is presented, showing the higher concentration of $S$. mansoni intermediate hosts, mainly B. glabrata, in three mesoregions - IV Metropolitana de Belo Horizonte, VI Norte de Minas and XI Vale do Rio Doce - with quite significant schistosomiasis prevalence rates.

B. glabrata was found naturally infected with $S$. mansoni in several municipalities and there were focuses with snail infection rates of $85 \%$ (Souza et al. 1997). In the mesoregions of Noroeste de Minas (V), Sul/Sudoeste de Minas (VIII) and Triângulo Mineiro/Alto Paranaíba (IX), where B. glabrata is rarely found, the occurrence of the parasitosis is very low (Figure). Although B. tenagophila is not widely spread in Minas Gerais, it was found naturally infected with $S$. mansoni in the municipalities of Jaboticatubas, Itajubá, Belo Horizonte and Ouro Branco (Melo \& Pereira 1985, Carvalho et al. 1985a,b, Silva et al. 1994), localized in the mesoregions Metropolitana de Belo Horizonte (IV) and Sul/Sudoeste de Minas (VIII). The natural infection rates of this species in those focuses are low, ranging from 0.03 to $17.5 \%$. B. straminea was mainly found in three mesoregions, Metropolitana de Belo Horizonte (IV), Noroeste de Minas (V) and Norte de Minas (VI). This species has not been found naturally infected with $S$. mansoni in Minas Gerais, as well as B. peregrina, but they have been experimentally infected with $S$. mansoni (Paraense 1973, Souza et al. 1981a,b, 1983, Souza 1986). B. intermedia was found only in the Triângulo Mineiro/Alto Paranaíba (IX) mesoregion, in the boundaries with the states of Mato Grosso do Sul and São Paulo, where its occurrence had already been recorded. $B$. occidentalis, resistant to $S$. mansoni infection and very similar to $B$. tenagophila by the morphology of the shell, was found in two municipalities (Table).

The current geographic distribution shows a high concentration of Biomphalaria species up to six per mesoregions, in Minas Gerais (Figure).

Studies on the planorbid fauna in all states of Brazil, mainly in less investigated regions, should be motivated with the objective to better know the geographic distribution of snail vectors of schistosomiasis and other helminthiases, aiming to control the risk of transmission.

\section{ACKNOWLEDGEMENTS}

To Dr Frederico Carlos Carvalho Soares, Regional Coordinator of Fundação Nacional de Saúde in Minas Gerais, for bibliography on snails of epidemiological importance in the transmission of schistosomiasis in the State of Minas Gerais.

\section{REFERENCES}

Andrade RM 1956. Nota sobre a campanha contra a esquistossomose mansoni em algumas localidades do Vale do Alto Rio Doce, Minas Gerais (Brasil). Rev Bras Malariol D Trop 8: 387-390.

Baker FC 1945. The Molluscan Family Planorbidae, University of Illinois Press, Urbana, $530 \mathrm{pp}$.

Barbosa FS, Dobbin Júnior JE 1951. Estrutura interna dos Australorbis (Mollusca, Planorbidae) de Pernambuco e outros estados. Publicações Avulsas do Instituto Aggeu Magalhães 1: 1-8.

Barbosa FS, Barbosa I, Carneiro E 1958. Morfologia de Australorbis nigricans (Spix) e suas relações com Australorbis glabratus (Say). Ciênc Cult 10: 144.

Barros JR 1934. Schistosomiasis mansoni. Rev Med Minas 1: 25-43.

Basseres MS, Pantoja WP 1947. Esquistossomose; prevalência de $S$. mansoni em planorbideos. Rev Serv Espec Saúde Pública 1: 149-162.

Brener Z, Mourão OG 1956. Inquéritos clínicoepidemiológicos em focos endêmicos de esquistossomose mansoni em Minas Gerais. Rev Bras Malariol D Trop 3: 519-526.

Carvalho OS, Guimarães CT, Massara CL, Bonesio JER 1985a. Situação atual da esquistossomose mansoni no lago da Pampulha, Belo Horizonte, MG, Brasil. Rev Saúde Pública 19: 270-277.

Carvalho OS, Massara CL, Guerra HL, Campos YR, Caldeira RL, Chaves A, Katz N 1998a. Re-evaluation of schistosomiasis mansoni in Minas Gerais Brazil III. Noroeste de Minas mesoregion. Rev Inst 
Med Trop S Paulo 40: 277-279

Carvalho OS, Massara CL, Rocha RS, Katz N 1989. Esquistossomose mansoni no Sudoeste do Estado de Minas Gerais (Brasil). Rev Saúde Pública 23: 341344.

Carvalho OS, Massara CL, Silveira Neto HV, Alvarenga AG, Vidigal THDA, Guerra HL, Santos MA, Chaves A, Katz N 1994. Schistosomiasis mansoni in the Region of the Triângulo Mineiro, State of Minas Gerais, Brazil. Mem Inst Oswaldo Cruz 89: 509512.

Carvalho OS, Massara CL, Silveira Neto HV, Guerra HL, Caldeira RL, Mendonça LF, Vidigal THDA, Chaves A, Katz N 1997. Re-evaluation of schistosomiasis mansoni in Minas Gerais, Brasil II. Alto Paranaíba Mesoregion. Mem Inst Oswaldo Cruz 92: 141-142.

Carvalho OS, Nunes IM, Caldeira RL 1998b. First report of Biomphalaria glabrata in the State of Rio Grande do Sul, Brazil. Mem Inst Oswaldo Cruz 93: 39-40.

Carvalho OS, Souza CP, Katz N 1985b. Primeiro encontro de Biomphalaria tenagophila (d'Orbigny, 1835) naturalmente infectada com Schistosoma mansoni, em Itajubá, sul do estado de Minas Gerais, Brasil. Rev Saúde Pública 19: 88-91.

Correa LR, Paraense WL 1971. Susceptibility of Biomphalaria amazonica to infection with two strains of Schistosoma mansoni. Rev Inst Med Trop S Paulo 13: 387-390.

Deslandes N 1951. Técnica de dissecção e exame de planorbídeos. Rev Serv Espec Saúde Pública 4: 371382.

Dias E 1953. Estudos preliminares sobre a esquistossomose mansoni no município de Bambuí, Estado de Minas Gerais. Rev Bras Malariol D Trop 5: 211215.

FNS - Fundação Nacional de Saúde 1995. Relatório de Atividades, Programa de Controle de Esquistossomose, 3 pp.

FNS - Fundação Nacional de Saúde 1998. Lista dos municípios do estado de Minas Gerais que apresentam planorbídeos de importância epidemiológica na transmissão da esquistossomose, Gerência Técnica de Esquistossomose de Brasília, 16 pp.

Freitas CA 1972. Situação atual da esquistossomose no Brasil. Rev Bras Malariol D Trop 24: 31-55.

Freitas JR 1978. Pesquisa sobre o habitat primitivo de planorbídeos no parque florestal do Rio Doce. Relatório Fundação de Desenvolvimento da Pesquisa da Universidade Federal de Minas Gerais, Belo Horizonte, $80 \mathrm{pp}$.

Freitas JR, Junqueira DV, Gerken SE 1972. Habitats primitivos de hospedeiros do $S$. mansoni na região de Lagoa Santa, MG. Ciênc Cult 24: 377.

IGA - Instituto de Geociências Aplicadas 1995. Relação das coordenadas e altitudes das sedes dos municípios e outras localidades do estado de Minas Gerais. Secretaria de Estado de Ciência e Tecnologia, Fundação Centro Tecnológico de Minas Gerais, 38 pp.

Katz N, Antunes CMF, Andrade RM, Pellegrino J, Coelho PMZ 1970. An attempt to control schistosomiasis mansoni in an endemic area by combining clinical treatment and molluscicide application. $J$ Parasit 56: 434.
Katz N, Peixoto SV 2000. Análise crítica da estimativa do número de portadores de esquistossomose mansoni no Brasil. Rev Soc Bras Med Trop 33: 303-308.

Lima LC, Soares DM, Guimarães CT 1993. Biomphalaria occidentalis Paraense, 1981 in the state of Minas Gerais, Brazil. Mem Inst Oswaldo Cruz 88: 289-292.

Lucena DT 1956. Resenha Sistemática dos Planorbídeos Brasileiros, Ed. Recife SA, Recife, 104 pp.

Martins AV 1938. Contribuição ao estudo do gênero Australorbis Pilsbry 1934. Mem Inst Biol Ezequiel Dias 2: 5-61.

Martins AV, Versiani W 1938. "Schistosomose mansoni" no norte de Minas Gerais, Brasil Méd 52: 812-816.

Martins AV, Martins G, Falcão AL 1953. Distribuição geográfica dos planorbídeos e seus índices de infestação pelas cercárias de Schistosoma mansoni no município de Belo Horizonte, Minas Gerais. $11^{\circ}$ Congresso Brasileiro de Higiene, Curitiba, 10 pp.

Melo AL, Pereira LH 1985. On the finding of Biomphalaria tenagophila naturally infected with Schistosoma mansoni in the State of Minas Gerais, Brazil. Rev Inst Med Trop S Paulo 27: 99-101.

Oliveira MP, Castro GA 1979. Adenda ao Ensaio de Catálogo de Moluscos do Brasil de Frederico Lange de Morretes. Comunicações Malacológicas n. 11. Bol Inst $\mathrm{Ci}$ Biol Geoci 26, Juiz de Fora, 8 pp.

Paraense WL 1955. Autofecundação e fecundação cruzada em Australorbis glabratus. Mem Inst Oswaldo Cruz 53: 276-284.

Paraense WL 1958. The genera "Australorbis", "Tropicorbis", "Biomphalaria", "Platytaphius" and "Taphius". (Pulmonata, Planorbidae). Rev Bras Biol 18: 65-80.

Paraense WL 1966. The synonymy and distribution of Biomphalaria peregrina in Neotropical Region. Rev Bras Biol 26: 269-296.

Paraense WL 1972. Fauna planorbídica do Brasil. In CS Lacaz, GR Baruzzi, WJR Siqueira (eds), Introdução à Geografia Médica do Brasil, Edgard Blucher \& USP, São Paulo, p. 213-239.

Paraense WL 1973. Susceptibility of Biomphalaria peregrina from Brazil and Ecuador to two strains of Schistosoma mansoni. Rev Inst Med Trop S Paulo 15: 127-130.

Paraense WL 1975. Estado atual da sistemática dos planorbídeos brasileiros. Arq Mus Nac 55: 105-111.

Paraense WL 1981. Biomphalaria occidentalis sp. n. from South America (Mollusca Basommatophora Pulmonata). Mem Inst Oswaldo Cruz 76: 199-211.

Paraense WL 1983. A survey of planorbid molluscs in the Amazonian region of Brazil. Mem Inst Oswaldo Cruz 78: 343-361.

Paraense WL 1986. Distribuição dos caramujos no Brasil. In FA Reis, I Faria, N Katz (eds), Modernos Conhecimentos sobre Esquistossomose Mansônica, Suplemento dos Anais 1983/84, vol. 14, Academia Mineira de Medicina, Belo Horizonte, p. 117-128.

Paraense WL, Deslandes N 1955a. Observations on the morphology of "Australorbis glabratus". Mem Inst Oswaldo Cruz 53: 87-103.

Paraense WL, Deslandes N 1955b. Observations on the morphology of Australorbis nigricans. Mem Inst 
Oswaldo Cruz 53: 121-134.

Paraense WL, Deslandes N 1956a. Observations on Australorbis janeirensis (Clessin, 1884). Rev Bras Biol 16: 81-102.

Paraense WL, Deslandes N 1956b. "Australorbis inflexus" sp. n. from Brazil (Pulmonata, Planorbidae). Rev Bras Biol 16: 149-158.

Paraense WL, Deslandes N 1959. The renal ridge as a reliable character for separating Taphius glabratus from Taphius tenagophilus. Am J Trop Med Hyg 8: 456-472.

Paraense WL, Santos JM 1953. Um ano de observações sobre esquistossomose em planorbídeos da Lagoa Santa. Rev Bras Malariol D Trop 3: 253-269.

Paraense WL, Alencar AJT, Correa LR 1983. Distribuição dos planorbídeos e prevalência da xistosomose mansoni no Estado do Espírito Santo. Mem Inst Oswaldo Cruz 78: 374-384.

Paraense WL, Fauran P, Courmes E 1964. Observations sur la morphologie, la taxonomie, la repartition geographique et les gîtes d'Australorbis schrammi. Bull Soc Pathol Exot 57: 1236-1254.

Paraense WL, Pereira O, Pinto DB 1954. Re-invasion of breading places by snails. Trans $R$ Soc Trop Med Hyg 48: 540.

Penido HM, Pinto DB, Deslandes N 1951. Estudo comparativo da anatomia interna de caramujos provenientes de Minas Gerais, Bahia, Pernambuco e Pará. Rev Serv Espec Saúde Pública 4: 383-405.

Pinto C 1944. Um ano de combate às doenças parasitárias que atacam os rodoviários da estrada Rio-Bahia, 1942 a 1943. Mem Inst Oswaldo Cruz 40: 210-340.

Pinto C, Almeida AF 1948. Schistosomiasis Mansoni no Brasil, Monografias do Inst Osvaldo Cruz 5, 287 pp.

Pinto C, Almeida AF 1954a. Epidemiologia da esquistossomíase mansoni no Brasil. Rev Brasil Med 2: $912-$ 918.

Pinto C, Almeida AF 1954b. Distribuição geográfica e frequência do Schistosoma mansoni no Brasil. Rev Brasil Med 2: 1000-1008.

Pinto DB, Deslandes N 1953. Contribuição ao estudo da sistemática de planorbídeos brasileiros. Rev Serv Espec Saúde Pública 6: 135-167.

Rey L 1991. Schistosoma e esquistossomose: epidemiologia e controle. In Parasitologia, Guanabara Koogan, Rio de Janeiro, p. 389-410.

Ruiz JM 1951. Nota sobre a cercariofagia de um oligochaeta do gênero Chaetogaster V. Baer, 1827. An Fac Farm Odontol Univ São Paulo 9: 51-56.

Ruiz JM 1952. Índices cercáricos específicos do Schistosoma mansoni verificados em Neves e Mariana, estado de Minas Gerais. Mem Inst Butantan 24: 63-68.

Schlemper Junior BR, Ferreira Neto JA, São Thiago PT, Bressan C, Amarante AR 1996. Distribuição geográfica de planorbídeos em Santa Catarina, Brasil. Rev Soc Bras Med Trop 29: 411-418.

Silva RE, Melo AL, Pereira LH, Frederico LF 1994. Levantamento malacológico da bacia hidrográfica do lago Soledade, Ouro Branco (Minas Gerais, Brasil). Rev Inst Med Trop S Paulo 36: 437-444.

Souza CP 1986. Estudo de moluscos do gênero Biomphalaria de Minas Gerais, com relação a adaptação parasito hospedeiro e importância na epidemiologia da esquistossomose. Rev Inst Med Trop S Paulo 28: 287-292.

Souza CP, Lima LC 1990, 1997. Moluscos de Interesse Parasitológico do Brasil, Série Esquistossomose 1, $2^{a}$ ed., Fundação Oswaldo Cruz, Centro de Pesquisas René Rachou, Belo Horizonte, 79 pp.

Souza CP, Araújo N, Azevedo MLL 1983. Estudo da potencialidade de populações de Biomphalaria straminea do Estado de Minas Gerais, como hospedeiras do Schistosoma mansoni. Rev Inst Med Trop S Paulo 78: 251-256.

Souza CP, Drummond SC, Silva CJE, Queiroz LA, Guimarães CT, Rocha RS 1998a. Investigação sobre a transmissão da esquistossomose no complexo turístico da Serra do Cipó, MG. Informe Epidemiol SUS 4: 43-51.

Souza CP, Jannotti-Passos LK, Coura-Filho P 1997. Risk of transmission of schistosomiasis in the Parque Estadual do Rio Doce (PERD) MG, (Brazil) and its surroundings. II: Malacological Survey in Dionísio, MG. In VI International Simposium on Schistosomiasis, Belo Horizonte, p. 136.

Souza CP, Jannotti-Passos LK, Freitas JR 1995. Degree of host-parasite compatibility between Schistosoma mansoni and their intermediate molluscan hosts in Brazil. Mem Inst Oswaldo Cruz 90: 5-10.

Souza CP, Lima LC, Jannotti-Passos LK, Ferreira SS, Guimarães CT, Vieira IBF, Mariani-Junior R 1998b. Moluscos límnicos da microrregião de Belo Horizonte, MG, com ênfase nos vetores de parasitoses. Rev Soc Bras Med Trop 31: 449-456.

Souza CP, Pereira JP, Rodrigues MS 1981a. Atual distribuição geográfica dos moluscos hospedeiros intermediários do Schistosoma mansoni em Belo Horizonte, MG, Brasil. Mem Inst Oswaldo Cruz 76: 383-391.

Souza CP, Rodrigues MS, Azevedo MLL, Araújo N 1981b. Suscetibilidade de populações de Biomphalaria straminea (Dunker, 1848) de Minas Gerais, à infecção por Schistosoma mansoni. Rev Inst Med Trop S Paulo 23: 212-216.

Teixeira MJ 1919. A schistosomose mansonica na infancia em Belo Horizonte, Thesis, Faculdade de Medicina, Imprensa Oficial, Belo Horizonte, 107 pp.

Teles HMS 1987. Distribuição de Biomphalaria straminea (Dunker, 1848) (Pulmonata, Planorbidae) no Estado de São Paulo, Brasil. Ciênc Cult 40: 508-512.

Teles HMS 1989. Distribuição de Biomphalaria tenagophila e B. occidentalis no Estado de São Paulo (Brasil). Rev Saúde Pública 23: 244-253.

Teles HMS 1996. Distribuição de Biomphalaria straminea ao sul da região neotropical, Brasil. Rev Saúde Pública 30: 341-349.

Teles HMS, Pereira PAC, Richinitti LMZ 1991. Distribuição de Biomphalaria (Gastropoda, Planorbidae) nos Estados do Rio Grande do Sul e Santa Catarina, Brasil. Rev Saúde Pública 25: 350352.

Vidigal THDA, Caldeira RL, Simpson AJG, Carvalho OS 2000. Further studies on the molecular systematics of Biomphalaria snails from Brazil. Mem Inst Oswaldo Cruz 95: 57-66. 